\title{
Small Informational Size and Interim Cores of Large Quasilinear Economies*
}

\author{
Yusuke Kamishiro $^{\dagger}$ Roberto Serrano ${ }^{\ddagger}$
}

August 2018

\begin{abstract}
We provide nonemptiness results of approximate interim cores with endogenous communication in large quasilinear economies, where every agent's informational size is small. We offer results for both replica and more general sequences of economies.

Keywords: Asymmetric Information; Large Quasilinear Economies; Informational Smallness; Approximate Interim Cores.
\end{abstract}

JEL Classification: C71, C72, D51, D82.

*Kamishiro gratefully acknowledges support from JSPS KAKENHI (JP16H03121).

${ }^{\dagger}$ Department of Economics, Kanto Gakuin University; ykamishi@kanto-gakuin.ac.jp

${ }^{\ddagger}$ Department of Economics, Brown University; roberto_serrano@brown.edu 


\section{Introduction}

In this paper we consider nonemptiness of the core in interim exchange economies with asymmetric information when agents' informational size is small (McLean and Postlewaite (2002)). Within this model of informational size, previous results are available for the $e x$ ante core (McLean and Postlewaite (2003, 2005)), but existence results for the interim core are missing. ${ }^{1}$ If coalitions are formed at the interim stage, we need to specify the information that agents in a coalition are allowed to use in constructing an objection. Depending on the extent of communication within the coalition, several interim core concepts have been proposed. The literature began with concepts where communication is exogenous, such as the coarse core and the fine core in Wilson (1978), but more recently, interim cores with endogenous communication have been made available; see Forges, Minelli, and Vohra (2002) or Forges and Serrano (2013) for surveys.

The current paper concentrates on a class of interim cores with endogenous information transmission, namely, the core with respect to (w.r.t.) equilibrium blocking, as termed in Kamishiro and Serrano (2011). Indeed, Serrano and Vohra (2007) show that several core notions previously proposed in the literature, such as the credible core in Dutta and Vohra (2005) or the virtual-utility core in Myerson (2007), can be unified into the notion of core w.r.t. equilibrium blocking (see Kamishiro and Serrano (2011) for a further elaboration).

In general, when incentive constraints are incorporated into core notions, it is difficult to obtain nonemptiness of interim cores of exchange economies, given the results in Vohra (1999) and Forges, Mertens, and Vohra (2002), which show that the incentive-compatible coarse core, a superset of all cores w.r.t equilibrium blocking, might be empty. This suggests the consideration of approximate cores, where the blocking constraints are somewhat relaxed.

Going in that direction, this paper considers large economies, and restricts attention to quasilinear preferences. Assumptions made on the information structure will in general matter for the results one can obtain. For instance, Kamishiro, Serrano, and Wooders (2015) consider the case where there is a small number of informed agents and an unbounded number of uninformed agents. In fact, the bulk of their argument

\footnotetext{
${ }^{1}$ However, Kamishiro and Serrano (2011) study the convergence question in this model in their Section 5.2 .
} 
is built around economies with exclusive information, in which there are finitely-many monopolists of different pieces of information. The paper shows that, in large quasilinear economies, the "small group effectiveness" condition suffices to obtain nonemptiness of approximate interim cores w.r.t. equilibrium blocking. In contrast, it also shows that the exact incentive-compatible coarse core might still be empty.

The current paper analyzes the opposite case, in the sense that all agents can have private information and all of them are informationally small, in the sense of McLean and Postlewaite (2002). Roughly speaking, agents are informationally small if, with high probability, agents are not able to change the estimate that others have of the likelihood of the state by misreporting their signals. In such scenarios, we show nonemptiness of the approximate interim cores w.r.t. equilibrium blocking, without relying on "small group effectiveness." Theorem 3.2 shows the result for replica sequences of economies, in which the number of copies of each agent type is the same, and Theorem 3.3 extends it to more general sequences.

In order to obtain nonemptiness of approximate interim cores, we mainly rely on the results of Dutta and Vohra (2005) and Kamishiro and Serrano (2011). These results establish that having an ex post core allocation that is incentive compatible is sufficient in quasilinear economies to show that the core w.r.t. equilibrium blocking be nonempty. This is the reason to restrict attention to quasilinear preferences, and we do not know whether the argument extends to more general settings. In addition, a variant of a lemma in McLean and Postlewaite (2002), adapted to our model, will be key to complete our proof, by allowing us to construct an incentive-compatible rule that lies in the approximate ex post core.

This brief paper proceeds as follows: while section 2 provides the basic framework and definitions, and briefly reviews the notions of informational size in McLean and Postlewaite (2002), section 3 offers nonemptiness results of the approximate interim core.

\section{The Model}

We consider sequences of economies with the property that all agents are informationally small in the sense of McLean and Postlewaite (2002). These authors consider a model in which the agents' utility functions depend on an underlying but unobserved state of nature, and in which each agent receives a private signal that is correlated with the state. 


\subsection{Notation and Definitions}

We begin with a few preliminaries in order to define a pre-economy and an economy. Let $N=\{1, \ldots, n\}$ denote a fixed finite set of agent types. We denote a (finite) state space as $\Theta=\left\{\theta_{1}, \ldots, \theta_{m}\right\}$. In this model, nature chooses an element $\theta \in \Theta$. The state of nature is unobservable, but each agent type $i$ receives a "signal" that is correlated with nature's choice of $\theta$. Let $T_{1}, \ldots, T_{n}$ be finite sets, where $T_{i}$ represents the set of possible signals that agent type $i \in N$ might receive and let $T=\prod_{i} T_{i}$. We take as given a probability distribution, denoted by $P$, on $\Theta \times T$. We assume that for every $\theta$, $\theta^{\prime}$ with $\theta \neq \theta^{\prime}$, there exists a $t \in T$ such that $P(t \mid \theta) \neq P\left(t \mid \theta^{\prime}\right)$.

The consumption set of each agent type, denoted by $X_{i}$, is $R_{+}^{L-1} \times R$ and for each $\theta \in \Theta, e_{i} \in X_{i}$ denotes the (state-independent) endowment of agent type $i$ in state $\theta \in \Theta$. The preferences of agent type $i$ are represented by a utility function $u_{i}: R_{+}^{L-1} \times R \times \Theta \rightarrow R$ where $u_{i}\left(x_{i}, \theta\right)=v_{i}\left(x_{i}^{-L}, \theta\right)+x_{i}^{L}$ is the utility function of agent type $i$ in state $\theta$. We note that in this model agents' utility functions do not depend on $T$, but do depend on the state of nature.

The collection $\mathcal{E}=\left(\left\{u_{i}, X_{i}, e_{i}, T_{i}\right\}_{i \in N}, \Theta, P\right)$ will be called a private information preeconomy. And for each state $\theta \in \Theta$, the collection $\left\{u_{i}(\cdot, \theta), e_{i}\right\}_{i \in N}$ defines an associated (complete information) pre-economy.

A profile on $N$ is a function $f$ from $N$ to $Z_{+}$, the nonnegative integers; we interpret $f(i)$ as the number of agents with the same type. Given the set of agent types $N$ and a profile $f$, we denote the agent set as $\mathbf{N}$, i.e., $\mathbf{N}=\{(i, j): i=1, \ldots, n ; j=1, \ldots, f(i)\}$. Let $\|f\|$ denote the number of agents in a group represented by a profile $f$, i.e., $\|f\|=\sum_{i \in N} f(i)$.

We begin by considering exact replicas; that is, sequences of economies where the $r^{\text {th }}$ economy has $r$ agents of each type. This yields simpler mathematical expressions and more transparent arguments. However, we are able to extend our results for the case of more general sequences, as we explain below. That is, we begin by considering only profiles $f$ of agent types such that $f(i)=f\left(i^{\prime}\right)$ for all $i, i^{\prime} \in N$.

Given a pre-economy $\mathcal{E}$ and a profile $f$, let

$$
\mathcal{E}^{f}=\left(\left\{u_{(i, j)}, X_{(i, j)}, e_{(i, j)}, T_{(i, j)}\right\}_{i \in N, j=1, \ldots, r}, \Theta, P^{f}\right)
$$

be a private information economy, with $\|f\|(=r \times n$ agents, for some integer $r$, satisfying the following conditions. (Hereafter we denote $T^{f}:=\prod_{i \in N, j=1, \ldots, r} T_{(i, j)}$.) 
(1) $e_{(i, j)}=e_{i}$ and $X_{(i, j)}=X_{i}$ for all $i \in N$ and all $j=1, \ldots, r$,

(2) $u_{(i, j)}(x, \theta)=u_{i}(x, \theta)$ for all $x \in R_{+}^{L-1} \times R, i \in N$ and $j=1, \ldots, r$.

(3) $T_{(i, j)}=T_{i}$ for all $i \in N$ and $j=1, \ldots, r$.

(4) $P^{f}$ is a probability distribution on $\Theta \times T^{f}$ satisfying the following ${ }^{2}$ :

(a) For each $j=1,2, \ldots, r$, and each $\left(\theta, t_{1}, \ldots, t_{n}\right) \in \Theta \times T$,

$$
P\left(\theta, t_{(1, j)}, t_{(2, j)}, \ldots, t_{(n, j)}\right)=P\left(\theta, t_{1}, t_{2}, \ldots, t_{n}\right)
$$

(b) for each $\theta$, the probability distributions over

$$
\left(T_{(1,1)}, T_{(2,1)}, \ldots, T_{(n, 1)}\right), \ldots,\left(T_{(1, r)}, T_{(2, r)}, \ldots, T_{(n, r)}\right)
$$

are independent given $\theta$.

Thus, $\mathcal{E}^{f}$ is a private information economy with $\|f\|$ agents, containing $r$ copies of each agent type $i \in N$. With the standard nonredundancy assumption on the probability distributions, by the law of large numbers, it follows that, if $r$ is sufficiently large for all $i$, and if one could pool all signals, then one could assign a probability to each state that is arbitrarily close to 1 . We note that no agent's information is redundant in this process: regardless of the number of copies, each agent still has information that cannot be inferred from the aggregate information of other agents.

\subsection{Core Notions}

Let $\mathcal{E}^{f}$ be a private information economy. For each $S \subseteq \mathbf{N}, S$-feasible allocations for the economy are of the form $z: T_{S} \rightarrow \mathcal{A}_{S}$, where $\mathcal{A}_{S}=\left\{\left(\xi_{i}\right)_{i \in S} \mid \xi_{i} \in R_{+}^{L-1} \times R, \sum_{i \in S} \xi_{i} \leq\right.$ $\left.\sum_{i \in S} e_{i}\right\}$.

For ease of notation, we often denote agent $(i, j)$ simply by $k$. An $S$-feasible allocation $\left(z_{k}\right)_{k \in S}$ is incentive compatible if

$$
\begin{aligned}
\sum_{\theta \in \Theta} \sum_{t_{S \backslash\{k\}} \in T_{S \backslash\{k\}}} u_{k}\left(z_{k}\left(t_{S \backslash\{k\}}, t_{k}\right), \theta\right) P^{f} & \left(\theta, t_{S \backslash\{k\}} \mid t_{k}\right) \\
& \geq \sum_{\theta \in \Theta} \sum_{t_{S \backslash\{k\}} \in T_{S \backslash\{k\}}} u_{k}\left(z_{k}\left(t_{S \backslash\{k\}}, t_{k}^{\prime}\right), \theta\right) P^{f}\left(\theta, t_{S \backslash\{k\}} \mid t_{i}\right)
\end{aligned}
$$

for each $t_{k}, t_{k}^{\prime} \in T_{k}$ and $k \in S$.

\footnotetext{
${ }^{2}$ These sequences are called conditionally independent replicas in McLean and Postlewaite (2002).
} 
An incentive compatible allocation $z$ for $\mathbf{N}$ is in the core with respect to (w.r.t.) equilibrium blocking of some class of coalitional communication mechanisms whenever there does not exist an equilibrium rejection of $z$, i.e., a random blocking plan against $z$, for a communication mechanism in that class; see Kamishiro and Serrano (2011) for details.

Particular cases of this definition are the following:

- The incentive-compatible coarse core (Vohra (1999)) corresponds to the core of no communication mechanism.

- The credible core (Dutta and Vohra (2005)) corresponds to the core of deterministic mechanisms.

- The virtual-utility core (Myerson (2007)) corresponds to the core of measurable random mechanisms.

- The randomized mediated core (Serrano and Vohra (2007)) corresponds to the core of unrestricted random mechanisms.

The larger the class of communication mechanisms considered, the smaller the corresponding core. It follows that the core notions just described are therefore nested, the latter one being the smallest.

Coalition $S$ is said to have an ex post objection to a feasible allocation $z$ for $\mathbf{N}$ if there exist $y_{S} \in \mathcal{A}_{S}$ and $t^{f} \in T^{f}$ such that

$$
\sum_{\theta \in \Theta} P^{f}\left(\theta \mid t^{f}\right) u_{k}\left(y_{k}^{S}, \theta\right)>\sum_{\theta \in \Theta} P^{f}\left(\theta \mid t^{f}\right) u_{k}\left(z_{k}\left(t^{f}\right), \theta\right)
$$

for all $k \in S .^{3}$ The ex post core is the set of allocations to which there is no ex post objection.

The inclusion of the ex post core allocations satisfying incentive compatibility in the core w.r.t. equilibrium blocking of unrestricted random communication mechanisms was shown in Kamishiro and Serrano (2011, Proposition 3.1), which follows an argument in Dutta and Vohra (2005, Proposition 5.1) for the credible core.

\footnotetext{
${ }^{3}$ Our use of the term ex post refers to events that occur after the realization of the signal profile $t$, but before the realization of the state $\theta$ (allocations can depend on agents' types but not on $\theta$, which is assumed to be unobservable.
} 
Given $\varepsilon>0$, coalition $S$ is said to have an $\varepsilon$-ex post objection to $z$ if there exist $y_{S} \in \mathcal{A}_{S}$ and $t^{f} \in T^{f}$ such that

$$
\sum_{\theta \in \Theta} P^{f}\left(\theta \mid t^{f}\right) u_{k}\left(y_{k}^{S}, \theta\right)>\sum_{\theta \in \Theta} P^{f}\left(\theta \mid t^{f}\right) u_{k}\left(z_{k}\left(t^{f}\right), \theta\right)+\varepsilon
$$

for all $k \in S$. The $\varepsilon$-ex post core (or approximate ex post core) is the set of all allocations to which no coalition has an $\varepsilon$-ex post objection. The definitions of approximate or $\varepsilon$ interim blocking and its associated cores combine the definitions of $\varepsilon$-blocking and interim cores in the obvious way, and are therefore omitted.

\subsection{Definitions on Informational Size}

For the private information economy $\mathcal{E}^{f}$, we define next the notions of informational size, aggregate uncertainty, and distributional variability, originally introduced in McLean and Postlewaite (2002), as follows:

1. Informational size:

Let $P_{\Theta}^{f}$ be the conditional probability distribution on $\Theta$ given $t^{f} \in T^{f}$. Any array of agents' types $t^{f}=\left(t_{-k}, t_{k}\right) \in T^{f}$ induces a conditional distribution on $\Theta$ and, if agent $k$ unilaterally changes his announced signal from $t_{k}$ to $t_{k}^{\prime}$, this conditional distribution in general will also change. We consider agent $k$ to be informationally small if, for each $t_{k}$, there is a small probability assigned to the event that he can induce a large change in the conditional distribution on $\Theta$ by changing his announced signal from $t_{k}$ to some other $t_{k}^{\prime}$. McLean and Postlewaite (2002) formalized this in the following definition.

Let

$$
I_{\varepsilon}^{k}\left(t_{k}^{\prime}, t_{k}\right):=\left\{t_{-k} \in T_{-k} \mid\left\|P_{\Theta}^{f}\left(\cdot \mid t_{-k}, t_{k}\right)-P_{\Theta}^{f}\left(\cdot \mid t_{-k}, t_{k}^{\prime}\right)\right\|>\varepsilon\right\},
$$

where $\|\cdot\|$ is the 1 -norm.

The informational size of agent $k$ is defined as

$$
\nu_{k}^{P^{f}}=\max _{t_{k} \in T_{k}} \max _{t_{k}^{\prime} \in T_{k}} \min \left\{\varepsilon \geq 0 \mid \operatorname{Pr}\left\{\tilde{t}_{-k}^{\sim} \in I_{\varepsilon}^{k}\left(t_{k}^{\prime}, t_{k}\right) \mid \tilde{t_{k}}=t_{k}\right\} \leq \varepsilon\right\} .
$$

Loosely speaking, we will say that agent $k$ is informationally small with respect to $P^{f}$ if his informational size $\nu_{k}^{P^{f}}$ is small. That is, if agent $k$ receives signal $t_{k}$ 
but reports $t_{k}^{\prime} \neq t_{k}$, then the effect of this misreport is a change in the conditional distribution on $\theta$ from $P_{\Theta}^{f}\left(\cdot \mid t_{-k}, t_{k}\right)$ to $P_{\Theta}^{f}\left(\cdot \mid t_{-k}, t_{k}^{\prime}\right)$. If $t_{-k} \in I_{\varepsilon}^{k}\left(t_{k}^{\prime}, t_{k}\right)$ then this change is large in the sense that $\left\|P_{\Theta}^{f}\left(\cdot \mid t_{-k}, t_{k}\right)-P_{\Theta}^{f}\left(\cdot \mid t_{-k}, t_{k}^{\prime}\right)\right\|>\varepsilon$. Therefore, $\operatorname{Pr}\left\{\tilde{t}_{-k}^{\sim} \in I_{\varepsilon}^{k}\left(t_{k}^{\prime}, t_{k}\right) \mid \tilde{t_{k}}=t_{k}\right\}$ is the probability that $k$ can have a large influence on the conditional distribution on $\Theta$ by reporting $t_{k}^{\prime}$ instead of $t_{k}$ when his observed signal is $t_{k}$. An agent is informationally small if for each of his possible signals $t_{k}$, he assigns small probability to the event that he can have a large influence on the distribution $P_{\Theta}^{f}\left(\cdot \mid t_{-k}, t_{k}\right)$, given his observed signal.

2. Negligible aggregate uncertainty:

Next, aggregate uncertainty can be quantified. Let

$$
\mu_{k}^{P^{f}}:=\max _{t_{k} \in T_{k}} \min \left\{\varepsilon \mid \operatorname{Pr}\left\{\left\|P_{\Theta}^{f}\left(\cdot \mid t^{f}\right)-I_{\theta}\right\|>\varepsilon \text { for all } \theta \in \Theta \mid \tilde{t_{k}}=t_{k}\right\} \leq \varepsilon\right\},
$$

where $I_{\theta}$ denotes the measure that puts all its mass on state $\theta$.

If $\mu_{k}^{P^{f}}$ is small for each $k$, then we will say that $P^{f}$ exhibits negligible aggregate uncertainty. In this case, each agent knows that, conditional on his own signal, the aggregate information of all agents will, with high probability, provide a good prediction of the true state.

3. Distributional variability:

To define the measure of variability, we first define a metric $d$ on the simplex $\Delta_{\Theta}$ as follows: for each $\alpha, \beta \in \Delta_{\Theta}$, let

$$
d(\alpha, \beta):=\left\|\frac{\alpha}{\|\alpha\|_{2}}-\frac{\beta}{\|\beta\|_{2}}\right\|_{2},
$$

where $\|\cdot\|_{2}$ denotes the 2 -norm. Hence, $d(\alpha, \beta)$ measures the Euclidean distance between the normalizations of $\alpha$ and $\beta$. We define

$$
\Lambda_{k}^{P^{f}}=\min _{t_{k} \in T_{k}} \min _{t_{k}^{\prime} \in T_{k} \backslash\left\{t_{k}\right\}} d\left(P_{\Theta}^{f}\left(\cdot \mid t_{k}\right), P_{\Theta}^{f}\left(\cdot \mid t_{k}^{\prime}\right)\right)^{2} .
$$

This is a measure of the variability of the conditional distribution $P_{\Theta}^{f}\left(\cdot \mid t_{k}\right)$ as a function of $t_{k}$. 


\section{The Main Result}

Next, we present an important lemma for our main result:

Lemma 3.1. For each $\eta>0$ and each (complete information) feasible allocation $\mathcal{A}=$ $\left(\zeta_{i}\left(\theta_{1}\right), \ldots, \zeta_{i}\left(\theta_{m}\right)\right)_{i \in N}$ in each state in a pre-economy, there exists a number $\hat{r}$ such that: for all $r>\hat{r}$ and every profile $f$ satisfying $f(i)=r$ for all $i$ there exist an incentive compatible allocation $z(\cdot)$ for $\mathcal{E}^{f}$ and a collection $B_{1}, \ldots, B_{m}$ of disjoint subsets of $T^{f}$ such that

(i) $\operatorname{Pr}\left\{\tilde{t}^{f} \in \cup_{h=1}^{m} B_{h}\right\} \geq 1-\eta$,

(ii) $\operatorname{Pr}\left\{\tilde{\theta}=\theta_{h} \mid \tilde{t}^{f}=t^{f}\right\} \geq 1-\eta$ for all $h=1, \ldots, m$ and all $t^{f} \in B_{h}$,

(iii) for all agents $(i, j)$ in the profile $f$ and all $t^{f} \in B_{h}$,

$$
u_{(i, j)}\left(z_{(i, j)}\left(t^{f}\right) ; \theta_{h}\right) \geq u_{(i, j)}\left(\zeta_{i}\left(\theta_{h}\right), \theta_{h}\right)-\eta
$$

(iv) for all agents $(i, j)$ in the profile $f$ and all $t^{f} \in T^{f} \backslash\left[\cup_{h=1}^{m} B_{h}\right]$,

$$
z_{(i, j)}\left(t^{f}\right)=\hat{x}_{(i, j)}\left(t^{f}\right)
$$

where $\left(\hat{x}_{(i, j)}\left(t^{f}\right)\right)_{(i, j)}$ is an ex post core allocation in signal profile $t^{f}$ with agents' profile $f$.

Proof. By the Claim in McLean and Postlewaite (2002, page 2449), for every $\lambda>0$, there exists an integer $\hat{r}$ such that for every $r>\hat{r}$ and every profile $f$ satisfying $f(i)=r$ for all $i, \nu_{i, j}^{P^{f}} \leq \lambda$ and $\mu_{i, j}^{P^{f}} \leq \lambda$.

For a conditionally independent sequence, $P_{\Theta}^{f}\left(\cdot \mid t_{i, j}\right)=P_{\Theta}\left(\cdot \mid t_{i}\right)$ for every profile $f$ and all $t_{i, j} \in T_{i}$. In particular, $P_{\Theta}^{f}\left(\cdot \mid t_{i, j}\right)$ is independent of $r$ and it follows that $\Lambda_{i, j}^{P^{f}}=\Lambda_{i}^{P}$ for all $r$ and $j$. Furthermore, $\Lambda_{i}^{P}>0$.

Let $\mathcal{A}=\left(\zeta_{i}\left(\theta_{1}\right), \ldots, \zeta_{i}\left(\theta_{m}\right)\right)_{i \in N}$ be a (complete information) feasible allocation in each state $\theta_{h} \in \Theta$, and $\eta>0$. Let

$$
K_{1}:=\max _{\theta} \max _{i} v_{i}\left(\sum_{j=1}^{n} e_{j}^{-L}, \theta\right)+\sum_{j=1}^{n} e_{j}^{L} .
$$

Choose $\delta$ so that

$$
0<\delta<\min \left\{\frac{c(\eta, \mathcal{A})}{20 \sqrt{m} K_{1}}, \frac{\eta}{4}, \frac{1}{6}\right\},
$$

where $c(\eta, \mathcal{A})$ is the number given in Lemma A.1 in McLean and Postlewaite (2002) introduced below (we use this lemma later; we omit to write down the definition of the function $c$ since we will only directly use the lemma.) 
Define $\hat{\mu}^{P^{r}}=\max _{i, j} \mu_{i, j}^{P^{r}}, \hat{\nu}^{P^{r}}=\max _{i, j} \nu_{i, j}^{P^{r}}, \Lambda^{P^{r}}=\min _{i, j} \Lambda_{i, j}^{P^{r}}$, and $\Lambda^{P}=\min _{i} \Lambda_{i}^{P}$. Applying Steps 1 and 2 in McLean and Postlewaite (2002, page 2449), there exists an $\hat{r}$ such that for every $r>\hat{r}$ and every profile $f$ satisfying $f(i)=r, \hat{\nu}^{P^{f}} \leq \delta \Lambda^{P}=\delta \Lambda^{P^{f}}$, $\hat{\mu}^{P^{f}} \leq \delta \Lambda^{P}=\delta \Lambda^{P^{f}}$.

For each $h=1, \ldots, m$, let

$$
A_{h}^{f}=\left\{t^{f} \in T^{f} \mid\left\|P_{\Theta}^{f}\left(\cdot \mid t^{f}\right)-I_{\theta_{h}}\right\| \leq \hat{\mu}^{P^{f}}\right\}
$$

and

$$
B_{h}^{f}=\left\{t^{f} \in T^{f} \mid\left\|P_{\Theta}^{f}\left(\cdot \mid t^{f}\right)-I_{\theta_{h}}\right\| \leq \hat{\mu}^{P^{f}}+\hat{\nu}^{P^{f}}\right\},
$$

where we recall that $\|\cdot\|$ is the 1 -norm. Let

$$
A_{0}^{f}=T^{f} \backslash\left[\cup_{h} A_{h}^{f}\right] \text { and } \quad B_{0}^{f}=T^{f} \backslash\left[\cup_{h} B_{h}^{f}\right]
$$

We rely on the following:

Lemma (McLean and Postlewaite (2002, Lemma A.1)). Let $\mathcal{A}=\left(\zeta_{i}\left(\theta_{1}\right), \ldots, \zeta_{i}\left(\theta_{m}\right)\right)_{i \in N}$ be a (complete information) feasible allocation in each state $\theta_{h} \in \Theta$. For each $\eta \geq 0$, there exists a collection $\left\{\left\{y_{i}\left(t_{i}, \theta_{h}\right)\right\}_{\left(t_{i}, \theta_{h}\right) \in T_{i} \times \Theta}\right\}_{i \in N}$ satisfying:

(i) $y_{i}\left(t_{i}, \theta_{h}\right) \in R_{+}^{L}$ and $\sum_{i \in N}\left(y_{i}\left(t_{i}, \theta_{h}\right)-e_{i}\right) \leq 0$ for all $t_{i} \in T_{i}$ and all $\theta_{h} \in \Theta$,

(ii) $u_{i}\left(\zeta_{i}\left(\theta_{h}\right) ; \theta_{h}\right) \geq u_{i}\left(y_{i}\left(t_{i}, \theta_{h}\right)\right) \geq u_{i}\left(\zeta_{i}\left(\theta_{h}\right) ; \theta_{h}\right)-\eta$ for all $t_{i} \in T_{i}$ and all $\theta_{h} \in \Theta$,

(iii) for each $t_{i}, t_{i}^{\prime} \in T_{i}$ with $t_{i} \neq t_{i}^{\prime}$,

$$
\sum_{\theta_{h}}\left[u_{i}\left(y_{i}\left(t_{i}, \theta_{h}\right), \theta_{h}\right)-u_{i}\left(y_{i}\left(t_{i}^{\prime}, \theta_{h}\right), \theta_{h}\right)\right] P\left(\theta_{h} \mid t_{i}\right) \geq \frac{c(\eta, \mathcal{A})}{2 \sqrt{m}} \min _{i} \Lambda_{i}^{P} .
$$

Applying this lemma, there exists a collection $\left(\left\{y_{i}\left(t_{i}, \theta_{h}\right)\right\}_{\left(t_{i}, \theta_{h}\right) \in T_{i} \times \Theta}\right)_{i \in N}$ satisfying the above three conditions. In the private information economy, Let $z(\cdot)$ be the allocation defined as

$$
z_{i, j}\left(t^{f}\right):= \begin{cases}y_{i}\left(t_{i}, \theta_{h}\right) & \text { if } t^{f} \in B_{h}^{f}(h=1, \ldots, m) \text { and } t_{i, j}^{f}=t_{i}, \\ \hat{x}_{i, j}\left(t^{f}\right) & \text { if } t^{f} \in B_{0}^{f}\end{cases}
$$

where $\left(\hat{x}_{(i, j)}\left(t^{f}\right)\right)_{(i, j)}$ is an ex post core allocation in signal profile $t^{f}$ when agents' profile is $f$. The proof of the lemma can now be completed using exactly the same arguments as in Kamishiro (2011).

From this lemma, we can obtain the following nonemptiness result of the approximate core. 
Theorem 3.2. For every $\varepsilon>0$, there exists a number $\hat{r}$ such that: for all $r>\hat{r}$ and every profile $f$ satisfying $f(i)=r$ for all $i$, any $\varepsilon$-interim core w.r.t. equilibrium blocking of a private information economy $\mathcal{E}^{f}$ is nonempty.

Proof: Given $\varepsilon>0$, let

$$
K_{1}:=\max _{\theta} \max _{i} v_{i}\left(\sum_{i=1}^{n} e_{i}^{-L}, \theta\right)+\sum_{i=1}^{n} e_{i}^{L} \quad \text { and } \quad \eta:=\frac{\varepsilon}{1+K_{1}} .
$$

We pick a Walrasian equilibrium allocation for all the economies in a replication sequence, which, in the case of replicas, is simply an allocation for the basic economy $\left(\zeta_{i}\left(\theta_{1}\right), \ldots, \zeta_{i}\left(\theta_{m}\right)\right)_{i \in N}$ with one agent of each characteristic. Given $\eta>0$, we are able to choose $\hat{r}$ in accordance with the above lemma, such that for all $r>\hat{r}$ and every profile $f$ satisfying $f(i)=r$ for every $i$ there exists an incentive compatible allocation $z(\cdot)$ for $\mathcal{E}^{f}$ satisfying the conditions in the lemma.

From Dutta and Vohra (2005, Proposition 5.1) and Kamishiro and Serrano (2011, Proposition 3.1) it suffices to show that this allocation $z(\cdot)$ is not ex post $\varepsilon$-blocked by any coalition $S$. If $t^{f} \in T^{f} \backslash\left[\cup_{h=1}^{m} B_{h}\right]$, then there is no such blocking from condition (iv) in Lemma 3.1. So suppose that there exist $\left(y_{k}^{S}\right)_{k \in S} \in \mathcal{A}_{S}$ and $t^{f} \in B_{h}$ satisfying

$$
\sum_{k \in S} \sum_{\theta \in \Theta} u_{k}\left(y_{k}^{S}, \theta\right) P^{f}\left(\theta \mid t^{f}\right)>\sum_{k \in S} \sum_{\theta \in \Theta} u_{k}\left(z_{k}\left(t^{f}\right), \theta\right) P^{f}\left(\theta \mid t^{f}\right)+\varepsilon \cdot|S|
$$

We will show that this leads to a contradiction.

For each $t^{f} \in B_{h}$,

$$
\begin{aligned}
\sum_{k \in S} \sum_{\theta \in \Theta} u_{k}\left(z_{k}\left(t^{f}\right), \theta\right) P^{f}\left(\theta \mid t^{f}\right) & \geq(1-\eta) \sum_{k \in S} u_{k}\left(z_{k}\left(t^{f}\right), \theta_{h}\right) \\
& \geq(1-\eta) \sum_{k \in S}\left[u_{k}\left(\zeta_{k}\left(\theta_{h}\right), \theta_{h}\right)-\eta\right] \\
& >(1-\eta) \sum_{k \in S} u_{k}\left(\zeta_{k}\left(\theta_{h}\right), \theta_{h}\right)-\eta \cdot|S|
\end{aligned}
$$

On the other hand,

$$
\sum_{k \in S} \sum_{\theta} u_{k}\left(y_{k}^{S}, \theta\right) P^{f}\left(\theta \mid t^{f}\right) \leq(1-\eta) \sum_{k \in S} u_{k}\left(y_{k}^{S}, \theta_{h}\right)+\eta \cdot K_{1} \cdot|S|
$$


These imply that

$$
\begin{aligned}
(1-\eta) \sum_{k \in S} u_{k}\left(y_{k}^{S}, \theta_{h}\right)+\eta \cdot K_{1} \cdot|S| & \geq \sum_{k \in S} \sum_{\theta} u_{k}\left(y_{k}^{S}, \theta\right) P^{f}\left(\theta \mid t^{f}\right) \\
& >\sum_{k \in S} \sum_{\theta \in \Theta} u_{k}\left(z_{k}\left(t^{f}\right), \theta\right) P^{f}\left(\theta \mid t^{f}\right)+\varepsilon \cdot|S| \\
& >(1-\eta) \sum_{k \in S} u_{k}\left(\zeta_{k}\left(\theta_{h}\right), \theta_{h}\right)-\eta \cdot|S|+\varepsilon \cdot|S| .
\end{aligned}
$$

Thus, we conclude that

$$
\sum_{k \in S} u_{k}\left(y_{k}^{S}, \theta_{h}\right)>\sum_{k \in S} u_{k}\left(\zeta_{k}\left(\theta_{h}\right), \theta_{h}\right)
$$

contradicting the assumption that $\left(\zeta_{k}\left(\theta_{h}\right)\right)_{k \in N}$ is a Walrasian allocation of the associated economy in state $\theta_{h}$. Therefore, the allocation $z(\cdot)$ is not ex post $\varepsilon$-blocked by any coalition, i.e., it therefore must belong to the ex post $\varepsilon$-core of the $r \times n$ agents' economy.

Finally, we can extend our result to general nonreplica sequences, as follows:

Theorem 3.3. Given $g=\left(g_{1}, \ldots, g_{n}\right) \in Z_{+}^{n}$, for every $\varepsilon>0$, there exists a number $\hat{r}$ such that: for all $r>\hat{r}$ and every profile $f$ satisfying $f(i)=r g_{i}$ for all $i$, any $\varepsilon$-interim core w.r.t. equilibrium blocking of a private information economy $\mathcal{E}^{f}$ is nonempty.

This theorem clearly holds since we can regard an economy consisting of $g(i)$ copies of each characteristic $i$ as another original pre-economy.

Kovalenkov and Wooders (2003) define the $\varepsilon_{1}$-remainder $\varepsilon_{2}$-core in a complete information setting. Following their definition, the $\varepsilon_{1}$-remainder $\varepsilon_{2}$-core in our model is defined as an incentive compatible allocation in the $\varepsilon_{2}$ core of a sub-profile containing all but a fraction $\varepsilon_{1}$ of the agents. We can therefore state the following:

Corollary: For every $\varepsilon=\min \left\{\varepsilon_{1}, \varepsilon_{2}\right\}>0$, there exists a number $\hat{B}$ such that: for all $B>\hat{B}$ and every profile $f$ satisfying $\|f\| \geq B$, any $\varepsilon_{1}$-remainder $\varepsilon_{2}$-interim core w.r.t. equilibrium blocking of a private information economy $\mathcal{E}^{f}$ is nonempty.

In closing, a word about the technical differences with McLean and Postlewaite (2002) will be instructive. McLean and Postlewaite (2002) also show that, if the economy is sufficiently replicated, all agents are informationally small in the sense that the conditional distribution on the state of nature does not vary significantly in any agent's signal if 
other agents' signals are known. Moreover, they construct a mechanism that is incentive compatible, ex post individually rational, and nearly ex post Pareto efficient.

We have constructed an incentive compatible mechanism that is slightly different than the one considered by McLean and Postlewaite (2002) in Step 3 of their proof of Theorem 2. When the received signals are too weak to allow estimation of the true state with high probability, our mechanism assigns each agent an ex post core allocation. In contrast, McLean and Postlewaite (2002) constructs an incentive compatible mechanism that satisfies (i)-(iii) in the lemma above (for large $r$ ) but, when the received signals cannot estimate the true state with high probability, their mechanism assigns each agent their endowment. That is, the McLean and Postlewaite mechanism allocates each agent their endowment when, for some sets $B_{h}, t^{f} \in T^{f} \backslash\left[\cup_{h=1}^{m} B_{h}\right]$, where $B_{h}$ is the set of signal profiles such that the true state is estimated as $\theta_{h}$ with probability almost one.

As a consequence of their treatment of the case where signals are too weak, in general the McLean and Postlewaite (2002) mechanism does not yield an (approximate) ex post core allocation. If one tries to modify their rule directly so that it would yield an ex post core allocation for $t^{f} \in T^{f} \backslash\left[\cup_{h=1}^{m} B_{h}\right]$, then a key inequality of their proof that shows incentive compatibility breaks down. In order to overcome this problem, we have presented a variant of an argument in Kamishiro (2011), who takes a collection $B_{1}, \ldots, B_{m}$ of $T^{f}$ somewhat different from that in McLean and Postlewaite (2002). With Kamishiro's approach, for sufficiently large $r$, there exists an incentive compatible rule that yields an ex post core allocation when $t^{f} \in T^{f} \backslash\left[\cup_{h=1}^{m} B_{h}\right]$. (In order for the argument to go through, $r$ needs to be taken larger than in McLean and Postlewaite, 2002). To underscore the difference or our result here with Kamishiro (2011), we remark that his paper fixes the set of agents and assumes they have very accurate signals in order to establish nonemptiness of the exact coarse core, while we increase the number of agents and prove nonemptiness of approximate cores. With such a change, a result similar to McLean and Postlewaite's Step 3 in their proof of Theorem 2 has been obtained. (See also Lemma 4.5 in Kamishiro (2011)).

\section{References}

Dutta, B., Vohra, R., (2005). "Incomplete Information, Credibility and the Core," Mathematical Social Sciences 50, 148-165. 
Forges, F., Mertens, J.-F., Vohra, R., (2002). "The Ex Ante Incentive Compatible Core in the Absence of Wealth Effects," Econometrica 70, 1865-1892.

Forges, F., Minelli, E., Vohra, R., (2002). "Incentive and the Core of an Exchange Economy: a Survey," Journal of Mathematical Economics 38, 1-41.

Forges, F., Serrano, R., (2013). "Cooperative Games with Incomplete Information: Some Open Problems," International Game Theory Review 15, 301-317.

Kamishiro, Y., (2011). "Informational Size and the Incentive Compatible Coarse Core in Quasilinear Economies," Games and Economic Behavior 71, 513-520.

Kamishiro, Y., Serrano, R. (2011). "Equilibrium Blocking in Large Quasilinear Economies," Mathematics of Operations Research 36, 552-567.

Kamishiro, Y., Serrano, R., and Wooders, M. (2015), "Monopolists of Scarce Information and Small Group Effectiveness in Large Quasilinear Economies," 2018 revision of Working Paper 2015-11, Department of Economics, Brown University.

Kovalenkov, A., Wooders, M., (2003). "Approximate Cores of Games and Economies with Clubs," Journal of Economic Theory 110, 87-120.

McLean, R. P., Postlewaite, A., (2002). "Informational Size and Incentive Compatibility," Econometrica 70, 2421-2453.

McLean, R. P., Postlewaite, A., (2003). "Informational Size, Incentive Compatibility, and the Core of a Game with Incomplete Information," Games and Economic Behavior, 45, 222-241.

McLean, R., Postlewaite, A., (2005). "Core Convergence with Asymmetric Information," Games and Economic Behavior 50, 58-78.

Myerson, R. B., (2007). "Virtual Utility and the Core for Games with Incomplete Information," Journal of Economic Theory 136, 260-285.

Serrano, R., Vohra, R., (2007). "Information Transmission in Coalitional Voting Games," Journal of Economic Theory 134, 117-137.

Vohra, R., (1999). "Incomplete Information, Incentive Compatibility and the Core," Journal of Economic Theory 86, 123-147.

Wilson, R., (1978). "Information, Efficiency and the Core of an Economy," Econometrica 46, 807-816. 